\title{
NANOTECHNOLOGY IN ADHESIVE RESTORATIVE BIOMATERIALS
}

\author{
Dr. Carounanidy Usha, Dr. Bindu Meera John
}

\begin{abstract}
Adhesive restorative materials are tooth coloured materials that adhere to the enamel and dentin, either using the micro-mechanical retention or chemical bonding. Adhesion to tooth structure has obviated many of the disadvantages experienced with metallic materials providing better tooth-restorative margins, excellent aesthetics and ultra-conservation of tooth structure. Therefore, adhesive dentistry is considered as a vital breakthrough in restorative dentistry. If aesthetics and adhesions are their positive points, strength and wear resistance are their week points. In order to meet up to the standards of ideal bio mimetic material, exhaustive attempts are being made in rendering them as effective bioactive materials, by adding antibacterial property and remineralizing capacity. Nano technology, a revolution in science at large has played a pivotal role in overcoming the negative aspects of the adhesive restorative materials. This paper highlights few of the avenues where nanotechnology has effectively influenced the way the adhesive restorative materials perform.
\end{abstract}

Key words: adhesive dentistry, nano technology, nano fillers, antibacterial activity, remineralizing capacity

\section{Introduction:}

Restorations are artificial replacement of the lost tooth structure due to disease; it should restore the form, function of the tooth structure and also restore the aesthetics and phonetics of the patient. Various metallic and non-metallic materials are used for this purpose of restoration.

Restorative-tooth junction is a biologically week interface. Marginal degradation of the material results in micro leakage, percolation and microbial growth in the interface leading to secondary caries. Secondary caries is one of the major reason for restoration replacement. ${ }^{[1]}$ This is more evident with the metallic restorative materials. These materials are retained in the tooth preparation with macro mechanical retention that also necessitates excessive removal of healthy tooth structure.
Though the metallic restorations are strong and last long, the patients' acceptance is less due to their un aesthetic appearance.

Adhesive restorative materials are tooth coloured materials that adhere to the enamel and dentin, either using the micro-mechanical retention or chemical bonding. ${ }^{[2]}$ Adhesion to tooth structure has obviated many of the disadvantages experienced with metallic materials providing better toothrestorative margins, excellent aesthetics and ultraconservation of tooth structure. Therefore, adhesive dentistry is considered as a vital breakthrough in restorative dentistry.

Resin based composites and glass ionomer cements are two major class of tooth coloured adhesive restorative materials that are currently in use. While glass ionomer cement adheres to the tooth structure by chemical bonding, the composite

Dr. Carounanidy Usha, Professor; Dr. Bindu Meera John,Reader; Department of Conservative Dentistry and Endodontics, Indira Gandhi Intitute Dental Science, Sri Balaji Vidyapeeth, Puducherry 607402, India. 
resin restorative materials use acid etching of the tooth structure to adhere micro mechanically. ${ }^{[3,4]}$

Despite the numerous virtues of composite resins, strength, polymerization shrinkage and longevity have been their vices. Rigorous research is still going on to improve their mechanical properties, resulting in quick and effective succession of newer materials.

Yet another vital area of advancement is towards rendering them anti-cariogenic. Addition of antibacterial and/or remineralizing elements into their composition makes these materials bioactive, thus preventing dental caries around and underneath them. The release of such components to the immediate vicinity of the tooth is easier due to the dynamic and repairable bond evident in certain materials with chemical bonding. ${ }^{[5]}$

Nanotechnology, a major revolution in science, opens up new vistas in material science and other related technology, by providing 'plenty of room at the bottom', as stated by Richard P. Feynman. ${ }^{[6]}$ It is defined as the direct manipulation of materials at the nanoscale. It studies the properties of materials at nanoscale dimensions. Nanoscale dimension is less than the atomic size. One nanometre $(\mathrm{nm})$ equals 1 billionth of a meter. Nanomaterials behave very differently from the parent material. ${ }^{[7]}$ The physical, mechanical and biological properties exhibited are highly desirable in nanoscale. Thus nanomaterials have the power of creating new products or modify the properties of a material at the atomic/ molecular level.

Use of nanotechnology is already wide spread in other sciences, and in restorative material science addition of nanoparticles to the chemical composition of the materials is done with the intent of accentuating their properties in all dimensions. Especially, the adhesive restorative technology has effectively used nanotechnology in order to meet the ideal requirements of a restorative material.

\section{Nanotechnology in Dental Composite resin}

\section{Improvement in the mechanical properties}

Resin based composites are composed of a resin matrix reinforced with filler particles. The addition of filler particles to the composition is intended to improve the viscosity of the resin, to improve the strength and wear resistance, and also to improve the translucency. Silica is one of the major fillers used in composite resins. Various sizes and shapes of silica particles are added to generate different classes of composite resins such as macro filled (use of macro sized fillers of $50 \mu \mathrm{m}$ ), micro/ mini filled ( use of small/ micro sized fillers of 0.04 to $0.2 \mu \mathrm{m}$ ) and hybrid (use of a mixture of macro / $\mathrm{mini} / \mathrm{micro}$ fillers of 0.7 to $3.6 \mu \mathrm{m}$ ). Though the mechanical properties can be better with macro sized filler particles, the surface properties and polishability are better when the particle sizes are smaller. In addition it is impractical to load the matrix with larger size filler particles in higher volume percentage, which compromises on the matrix content. Hybrid composites that contained both macro and micro fillers had been the via media solutions for strength and aesthetics for a long time.

Recently fillers in nano sizes of 0.005 to 0.04 $\mu \mathrm{m}$ are added to composite resin and this new class is called as the Nanofilled composite resin. Barium boron fluoroalumino silicate glass, combination of aggregate zirconia/silicon cluster filler and silicon dioxide/fine particles of glass are some of the fillers used. The nanofillers used also varies in shape ranging from irregular to spherical. ${ }^{[8]}$ Nanohybrid composites are improvisation in this concept. This is similar to the hybrid concept, where nano sized particles along with micro filled particles are used as fillers. ${ }^{[9]}$

The addition of nano fillers result in the following enhancements in the restoration: ${ }^{[6,10]}$

1. Nano size of the fillers enable addition of more number of fillers thus increasing the surface area of fillers. Thus mechanical properties such as strength and abrasion resistance re improved.

2. Translucency also is improved due to the same reason. In addition, as the size of the fillers are smaller than the wavelength of the light, the optical property is enhanced.

3. Surface roughness of the composite resins, largely depend on the tendency of the filler particles to dislodge from the matrix. Polishing procedures tend to dislodge the filler particles. In contrast to large sized fillers, nano sized fillers even if they are dislodged, do not result in rough surface. However the nano clusters are linked to the matrix monomer at an atomic level, thus dislodgement may occur at 
all. This tendency to finish and polish the nano filled composites to a high gloss resulted in long lasting excellent aesthetics with reduced staining capacity.

4. A well-polished surface is also a poor substrate for bacterial adhesion and proliferation, thus tendency to cause biofilm related diseases such as dental caries and periodontal disease is less around the nano filled composite resins.

\section{Improvement in the antibacterial property}

Composite resin inherently do not possess any antibacterial effect. In fact studies have proven that metallic restorative materials like silver amalgam possess more antibacterial effect against Mutans streptococci than composite resin. Compounding to this disadvantage is the polymerization shrinkage. Conversion of the matrix monomer to polymer by polymerization process results in linear as well as volumetric shrinkage. The shrinkage stress generated can result in debonding at the toothrestoration interface leading to marginal leakage and secondary caries. Polymerization shrinkage is directly associated with the type and amount of the matrix present in the composite resin. Addition of higher volume percentage of the nano filler particles relatively reduces the matrix content, thus resulting in less polymerization shrinkage. Such resins form a class of low-shrinkage dental composites. ${ }^{[11]}$

In addition attempts have been made in introducing antibacterial elements in nano size to the composition to combat the cariogenic organisms. Broad spectrum antimicrobials such as metals and polymers have been used as nanoparticles.

Silver nanoparticles, zinc oxide nanoparticles and quaternary ammonium polyethylenimine nanoparticles are some used in the current generation of nanocomposites. ${ }^{[12]}$

\section{$\underline{\text { Silver nanoparticles }}$}

The antibacterial action of silver is attributed to the release of silver ions which interact with peptidoglycan cell wall and the plasma membrane. The effect is more in nano silver (NAg) that is directly proportionate to the high surface to volume ratio. NAg exhibits strong antibacterial activity against Mutans streptococci and Lactobacilli. Nanosilver particles in the size of $2.7 \mathrm{~nm}$ have shown high antibacterial efficacy. The small size of the particles enable easy diffusion of $\mathrm{Ag}$ ions into the complex dental biofilm and into the dentinal tubules. However, there can be a change in the aesthetics of the resin; therefore only $0.5-1 \%$ concentration has been recommended. ${ }^{[12,13]}$

\section{Zinc oxide nanoparticles}

These particles exhibit antibacterial effect on both gram positive and negative organisms. Its action is attributed to the production of $\mathrm{H}_{2} \mathrm{O}_{2}$ that inhibit the growth of planktonic organisms. It also releases zinc ions that inhibits biofilm formation and metabolism of sugar. But compared to nano silver, nano zinc is needed in higher concentration. Composite with $10 \%$ nanozinc has shown antibacterial action against mutans streptococci. ${ }^{[14,15]}$

\section{Quaternary ammonium polyetbylenimine nanoparticles (QAS-PEI)}

These cause bacterial lysis by binding to the cell membrane and cause cytoplasmic leakage. QAS salt containing polymers are immobilized in the polymer matrix to give long-lasting antibacterial property. But they can leach resulting in degradation of the mechanical properties over time. Thus they are incorporated into the composites as nanoparticles. $1 \%$ of QAS-PEI added as nano particles conferred anti-bacterial effect against S.mutans without altering the mechanical properties of the restorative materials. ${ }^{[16]}$

\section{Improcement in the remineralizing capacity}

Calcium, phosphate and fluorides play a major role in the remineralization-demineralization process of dental caries lesion formation. Tooth substance under acid attack releases these ions to the biofilm fluid and the saliva. This demineralization continues till the fluids are super saturated. The super saturated saliva then redeposit the mineral ions back to the tooth structure. A biomimetic material, should also behave in this way when used as restorative material. The chemical composition should ideally have calcium/ phosphate/ fluoride ions and leach them out on acidic attack to the nearby tooth structure assisting in tooth remineralization. They should also be capable of recharging themselves with remineralizing agents from the saliva. However such an ideal material is not present in the class of composite resins. Earlier attempts to incorporate 
fluorides into the resin composition was not successful. Despite their antibacterial effect and remineralizing capacity, their presence in the filling materials reduced the mechanical properties of the restoration. ${ }^{[17]}$

Recently fluoride is added as a nanomaterial in the form of Calcium fluoride. Addition of 20$30 \%$ calcium fluoride has been found to have high fluoride release without compromising on the mechanical features of the restoration. Such a release was found to be highly associated with acidic $\mathrm{pH}$ of 5.5. ${ }^{[18]}$ Calcium phosphate nanoparticles such as monocalcium phosphate monohydrate (MCPM) dicalcium phosphate anhydrous (DCPA) tetra calcium phosphate (TTCP) and amorphous calcium phosphate $(\mathrm{ACP})$ are also incorporated into the resin to confer remineralizing capacity. Nanoparticle Amorphous calcium phosphate (NACP) has been found to be effective in this regard as it is readily converted to crystallites. Nano-hydroxyapatite (N$\mathrm{HA}$ ) and nano-fluorhydroxyapaptite (N-FHA) are also evaluated for the same effect. ${ }^{[12]}$

\section{Nanotechnology in Glass Ionomer Cement}

Glass ionomer cements (GIC) belong to the group of acid based cements. Aluminosilicate glasses when mixed with polyacrylic acid produces a tooth coloured cement that can chemically bond to the calcium, phosphate of the mineral content of the tooth and also to the collagen of the organic content of the tooth. Introduction of this material paved way for minimally invasive dentistry, where no elaborate tooth preparation is required to accommodate. Interestingly GIC is considered cariogenic as it contains fluoride glasses that leach out fluoride ions to the tooth vicinity. It mimics the properties of dentin such as modulus of elasticity and insulating property, thus also called as artificial dentin. ${ }^{[19]}$

The major drawbacks, such as poor wear resistance and low strength are the ones that are being improvised with nanotechnology. Nanoparticulated ionomer is one such attempt. These are resin-modified glass ionomer cements with nanotechnology, which combine the benefits of RMGIC and bonded Nano filler particles in the range of 0.1 to 100 nanometers on the nanoscale. This broad range of filler particle is attributed to better strength, optical properties, and abrasion resistance of the Nanoparticulated ionomer. [20]
Also taking advantage of the fact that ions can readily travel in and out of the material, many antimicrobial additives such as nanoparticles of chlorhexidine hexametaphosphate (N-CHX HMP). These GICs released soluble CHX over a period of at least 33 days, and the quantity of $\mathrm{CHX}$ released was dependent on the doping of nanoparticles in the cement. ${ }^{21]}$ Other synthetic remineralizing agents such as nano-hydroxyapatite (N-HA) and nano-fluorhydroxyapaptite (NFHA) are also used in glass ionomer cement. The addition of $10 \%$ nanoHA (60-100 nm) to glass ionomer cement resulted in an increased resistance to demineralization and acceptable bonding strength compared with microHA added to glass ionomer cement. ${ }^{[12]}$

\section{Conclusion:}

Recent advances in adhesive technology point towards a constant and persistent attempts to further enhance the strengths of the adhesive restorative material and at the same time address and overcome the weaknesses. If aesthetics and adhesions are their positive points, strength and wear resistance are their week points. In order to meet up to the standards of ideal bio mimetic material, exhaustive attempts are being made in rendering them as effective bio active materials, by adding antibacterial property and remineralizing capacity. Such attempts that were not entirely successful earlier, currently are being realised in adhesive technology only with the introduction of nanotechnology in this field. The properties of any material is superior in its nano dimensions and with this has emerged unlimited possibilities in adhesive technology.

\section{References}

1. Mjör IA. Clinical diagnosis of recurrent caries. J Am Dent Assoc. 2005;136(10):1426-33.

2. Kugel G. Direct and indirect adhesive restorative materials: a review. Am J Dent. 2000;13(Spec No):35D-40D.

3. Francisconi LF, Scaffa PM, de Barros VR, Coutinho M, Francisconi PA. Glass ionomer cements and their role in the restoration of non-carious cervical lesions. J Appl Oral Sci. 2009;17(5):364-9.

4. Hervás-García A, Martínez-Lozano MA, Cabanes-Vila J, Barjau-Escribano A, Fos-Galve P. Composite resins. A review of the materials and clinical indications. Med Oral Patol Oral Cir Bucal. 2006;11(2):E215-20.

5. MJ Tyas, MF Burrow. Adhesive restorative materials: A review. Australian Dental Journal 2004;49:(3):112-121

6. Sule Tugba Ozak, Pelin Ozkan. Nanotechnology and dentistry. Eur J Dent. 2013; 7(1): 145-151. 
7. Freitas RA Jr. Nanodentistry. J Am Dent Assoc. 2000; 131(11):1559-65.

8. Mota EG, Hörlle L, Oshima HM, Hirakata LM. Evaluation of inorganic particles of composite resins with nanofiller content. Stomatologija, Baltic Dental and Maxillofacial Journal $2012 ; 14$ : 103-7,

9. de Moraes RR, Gonçalves Lde S, Lancellotti AC, Consani S, Correr-Sobrinho L, Sinhoreti MA. Nanohybrid resin composites: nanofiller loaded materials or traditional microhybrid resins? Oper Dent. 2009;34(5):551-7.

10. Chen MH. Update on dental nanocomposites. J Dent Res. 2010;89(6):549-60.

11. Pitel ML. Low-shrink composite resins: a review of their history, strategies for managing shrinkage, and clinical significance. Compend Contin Educ Dent. 2013;34(8):57890.

12. Melo MA1, Guedes SF, Xu HH, Rodrigues LK. Nanotechnology-based restorative materials for dental caries management.Trends Biotechnol. 2013;31(8):459-67.

13. Yoshida K, Tanagawa M, Atsuta M Characterization and inhibitory effect of antibacterial dental resin composites incorporating silver-supported materials. J Biomed Mater Res. 1999; 47(4):516-22.

14. Hernández-Sierra JF, Ruiz F, Pena DC, Martínez-Gutiérrez F, Martínez AE, Guillén Ade J, Tapia-Pérez H, Castañón GM. The antimicrobial sensitivity of Streptococcus mutans to nanoparticles of silver, zinc oxide, and gold. Nanomedicine. 2008; 4(3):237-40.
15. Aydin Sevinç B, Hanley L Antibacterial activity of dental composites containing zinc oxide nanoparticles. J Biomed Mater Res B Appl Biomater. 2010; 94(1):22-31.

16. Imazato $S$, et al. Antibacterial resin monomers based on quaternary ammonium and their benefits in restorative dentistry. Jpn Dent Sci Rev. 2012;48:115-125.

17. Kirsten GA, Takahashi MK, Rached RN, Giannini M, Souza EM. Microhardness of dentin underneath fluoridereleasing adhesive systems subjected to cariogenic challenge and fluoride therapy. J Dent. 2010; 38(6):460-8.

18. Xu HH, Moreau JL, Sun L, Chow LC. Novel CaF(2) nanocomposite with high strength and fluoride ion release. J Dent Res. 2010; 89(7):739-45.

19. Khoroushi M, Keshani F. A review of glass-ionomers: From conventional glass-ionomer to bioactive glass-ionomer. Dent Res J. 2013; 10(4): 411-420

20. Konde S, Raj S, Jaiswal D. Clinical evaluation of a new art material: Nanoparticulated resin-modified glass ionomer cement. J Int Soc Prev Community Dent. 2012; 2(2): 42-47.

21. Hook ER, Owen OJ, Bellis CA, Holder JA, O'Sullivan DJ, Barbour ME.Development of a novel antimicrobial-releasing glass ionomer cement functionalized with chlorhexidine hexametaphosphate nanoparticles. J Nanobiotechnology. 2014;12:3. 